\title{
Tbx2 confers poor prognosis in glioblastoma and promotes temozolomide resistance with change of mitochondrial dynamics
}

This article was published in the following Dove Press journal:

OncoTargets and Therapy

20 February 2017

Number of times this article has been viewed

Fuxin Yi

Jianzhou Du

Weimin $\mathrm{Ni}$

Weixian Liu

Department of Neurosurgery, First Affiliated Hospital of Jinzhou Medical University, Jinzhou, People's Republic of China
Correspondence: Fuxin Yi

Department of Neurosurgery, First Affiliated Hospital of Jinzhou Medical University, Renmin Street 2, Guta

District, Jinzhou, Liaoning 121000 ,

People's Republic of China

Tel +86I594067 6888

Email yifuxinliaoyi@sina.com
Abstract: Tbx 2 is a cancer-related protein that was found to be overexpressed in several human malignancies. The present study aims to investigate the clinical significance and biological role of Tbx 2 in human astrocytoma. We examined its protein expression in 102 cases of astrocytoma tissues using immunohistochemical staining. Negative Tbx2 staining was observed in normal astrocytes, and positive nuclear staining was found in 41 out of 102 astrocytoma specimens. The rate of Tbx2 overexpression in pylocytic astrocytoma, diffuse astrocytoma, anaplastic astrocytoma, and glioblastoma multiform (GBM) were $0 \%, 26.1 \%, 40 \%$, and $52 \%$, respectively. Tbx2 overexpression correlated with poor prognosis in patients with astrocytoma or GBM. Tbx2 plasmid transfection was performed in A172 cells, and Tbx2 siRNA knockdown was carried out in U251 cells. Cell Counting Kit-8, cell cycle analysis, and matrigel invasion assay showed that Tbx2 overexpression upregulated cell proliferation, G1-S transition, and invasion, with corresponding change of cyclin D1, p21, and MMP 2 and 9. Importantly, we demonstrated that Tbx 2 reduced apoptosis and conferred resistance to temozolomide in GBM cell lines. Further experiments showed that $\mathrm{Tbx} 2$ could regulate mitochondrial fission/fusion balance. Western blot showed that Tbx2 overexpression reduced caspase 3 cleavage, while it induced Bcl-2 and p-Drp1 upregulation. In conclusion, our results indicated that Tbx 2 might serve as an indicator for poor prognosis and also be useful as an important therapeutic in human GBM, which inhibits apoptosis through regulation of mitochondrial function.

Keywords: Tbx2, immunohistochemistry, glioblastoma, mitochondrial function

\section{Introduction}

Astrocytoma is the most common malignant tumor in the central nervous system and can be divided into Grade I (pylocytic astrocytoma), Grade II (diffuse astrocytoma), Grade III (anaplastic astrocytoma), and Grade IV (glioblastoma). Despite improvements in areas such as earlier diagnosis, surgery, and chemotherapy, the prognosis for this malignancy remains poor. ${ }^{1}$ Development of chemoresistance plays a vital role in the progression and poor response of human astrocytoma, and identifying of related targets is an important task. ${ }^{2,3}$

Tbx2 is one of the T-box family transcription factors that are involved in various biological processes such as development and tumorigenesis. ${ }^{4,5} \mathrm{Tbx} 2$ overexpression has been found in several cancers including breast cancer, lung cancer, and colorectal cancer. ${ }^{6-8}$ Previous reports showed that Tbx2 overexpression correlated with poor patient prognosis, cancer cell proliferation, and invasion. ${ }^{7,8}$ However, the expression pattern and biological roles of Tbx2 in human astrocytoma have not been investigated. 
In the present study, we examined clinical significance of Tbx2 overexpression in human astrocytoma by immunohistochemistry. We also explored the effects of Tbx2 on astrocytoma cell proliferation, invasion, and temozolomide resistance. In addition, the potential underlying mechanism of action of Tbx2 in astrocytoma cells was explored.

\section{Materials and methods}

\section{Patients and specimens}

The study protocol was approved by the institutional reviewer board of First Affiliated Hospital of Jinzhou Medical University. Primary tumor specimens were obtained from 102 patients diagnosed with astrocytoma who underwent resection in the First Affiliated Hospital of Jinzhou Medical University between 2009 and 2015. Written informed consent for use of the tumor specimens was obtained from human study participants, or their next of kin if patients were under 16 years of age. Sections were stained with hematoxylin and eosin and evaluated according to the 2004 World Health Organization classification guidelines.

\section{Immunohistochemistry}

Four- $\mu \mathrm{m}$ tissue sections were prepared. Immunohistochemistry was performed using the Elivision plus kit purchased from Maixin (MaiXin, Fuzhou, People's Republic of China). Deparaffinization was performed using xylene. Sections were rehydrated with graded alcohol. Antigen retrieval was performed in citrate buffer $\left(\mathrm{pH}\right.$ 6.0) for 2 minutes. $\mathrm{H}_{2} \mathrm{O}_{2}$ was used to block the endogenous peroxidase. Normal goat serum was used to reduce nonspecific binding. Then, sections were incubated with Tbx2 polyclonal antibody (1:60; Sigma, St Louis, MO, USA) overnight at $4^{\circ} \mathrm{C}$. Rabbit immunoglobulin (at the same concentration as for the antigen-specific antibody) was used as a negative control. The staining was followed by incubation with polymer secondary antibodies. The peroxidase reaction was developed using DAB plus from Maixin (Maixin, Fuzhou, People's Republic of China). Counterstaining was done with hematoxylin, and the sections were dehydrated in alcohol before mounting. For evaluation of Tbx2 staining, we counted 400 tumor cells and calculated the intensity and percentage of positively stained cells. Tbx2 was scored as positive or high expression when a specimen showed $>15 \%$ of cancer cells staining positive for $\mathrm{Tbx} 2$ protein.

\section{Cell culture and transfection}

U251 and U87 cell lines were obtained from American Type Culture Collection (Manassas, VA, USA). The cells were cultured in Dulbecco's Modified Eagle's Medium (Invitrogen, Carlsbad, CA, USA) containing 10\% fetal calf serum (Invitrogen).
siGENOME Tbx2 siRNAs pool and negative siRNAs pool were obtained from Dharmacon (GE Healthcare, Piscataway, NJ, USA). siRNA was transfected with DharmaFECT1 (GE Healthcare). pCMV6 Tbx2 plasmid was obtained from Origene (Origene, Rockville, MA, USA). Transfection was performed in A172 cell line using Attractene reagent (Qiagen, Hilden, Germany). For each well of a 6-well plate, $0.2 \mathrm{nmol}$ siRNA/1.2 $\mu \mathrm{g}$ plasmid DNA was used for transfection.

\section{CCK8 assay}

For Cell Counting Kit-8 (CCK8) assay, 24 hours after plasmid or siRNA transfection, cells were plated in 96-well plates at a concentration of approximately 2,000 cells per well and cultured for the next 4 days. For evaluation of cell viability, CCK8 solution was added to each well and incubated for 4 hours in the incubator. Then the plates were measured spectrophotometrically at $490 \mathrm{~nm}$.

\section{Matrigel invasion assay}

Matrigel invasion assay was carried out using a 24-well Transwell chamber from Costar (Corning, NY, USA) coated with $20 \mu \mathrm{L}$ Matrigel with a dilution rate of 1:6 (BD Biosciences, San Jose, CA, USA). About 48 hours after the transfection, cells were trypsinized and transferred to the upper chamber with the serum and incubated for 18 hours. To the lower chamber was added medium supplemented with $10 \%$ serum. Noninvading cells were wiped away, and cells that had invaded through the filter were fixed with $4 \%$ paraformaldehyde and stained with hematoxylin.

\section{Western blot analysis}

Total protein from tissue and cells was extracted and quantified using the Bradford method. Samples were separated by sodium dodecyl sulfate-polyacrylamide gel electrophoresis and transferred to polyvinylidene fluoride membranes (Millipore, Billerica, MA, USA) and incubated overnight at $4^{\circ} \mathrm{C}$ with antibody against Tbx2 (1:1,000; Sigma), cyclin D1 (1:1,000; Cell Signaling Technology, Danvers, MA, USA), Bcl-2 (1:1,000; Cell Signaling Technology), cytochrome C (1:800; Cell Signaling Technology), MMP2 (1:1,000; Cell Signaling Technology), MMP9 (1:1,000; Cell Signaling Technology), caspase-3 (1:800; Cell Signaling Technology), cleaved caspase-3 (1:800; Cell Signaling Technology), p-Drp1 (1:600; Cell Signaling Technology), and GAPDH (1:2,500; Cell Signaling Technology). The membranes were incubated with secondary antibodies (Santa Cruz, Dallas, TX, USA) at room temperature for 2 hours. Then membranes were developed using ECL Western blotting substrate (Thermo Fisher Scientific, San Jose, CA, USA) 
and the bands detected using DNR Imaging System (DNR, Jerusalem, Israel).

\section{Quantitative real-time polymerase chain reaction}

Real-time (RT-PCR) was carried out using the SYBR Green master mix from ABI (ABI, Waltham, MA, USA) with a 7,500 Real-Time PCR System (ABI). A dissociation step was performed to generate melting curves to confirm the specificity of the amplification. Expression levels of the analyzed genes were normalized to the expression of $\beta$-actin. The fold change of gene expression was calculated by the $2^{-\Delta \Delta C}$ method. The sequences of the primer pairs are as follows: Tbx2 forward, 5'-GGCTTCAACATCCTAAACTCC-3'; Tbx2 reverse, 5'-AAGATCGACCAACAACCCGTTT-3'. $\beta$-actin forward, 5'-ATAGCACAGCCTGGATAGCAAC GTAC-3', $\beta$-actin reverse, $5^{\prime}$-CACCTTCTACAATGAGCT GCGTGTG-3'.

\section{Detection of mitochondrial DNA (mtDNA) content}

Relative mtDNA content was measured by examining $N D 1$ and $H B B$ expression. The single-copy $H B B$ gene was used as control. The ratio of mtDNA copy number to $H B B$ copy number was determined for each sample from standard curves. Then mtDNA/HBB was normalized to a calibrator DNA and the normalized $\mathrm{mtDNA} / H B B$ ratio was determined as the copy number. The primer sequences for the mitochondrial $N D 1$ and $H B B$ are as follows: $N D 1$ forward, 5'-CCCTAAAACCCgCCACATCT-3'; ND1 reverse, 5'-GAGCGATGGTGAGAGCTAAGGT-3'. $H B B$ forward, 5'-GTGCACCTGACTCCTGAGGAGA-3'; $H B B$ reverse, 5'-CCTTGATACCAACCTGCCCAG-3'.

\section{Matrigel invasion assay}

Matrigel invasion assay was performed using a Transwell chamber from Costar coated with $20 \mu \mathrm{L}$ Matrigel (1:6 dilution; BD Biosciences). Cells were transferred to the upper chamber with serum free medium. To the lower chamber was added medium with $15 \%$ serum. After 18 hours incubation, cells that had invaded through the filter were fixed and stained with hematoxylin.

\section{Flow cytometry and Annexin V/propidium iodide analysis}

For determination of cell cycle, cells were seeded into $6 \mathrm{~cm}$ tissue culture dishes. About 48 hours after transfection, cells were harvested, fixed in 1\% paraformaldehyde, washed with phosphate-buffered saline (PBS), and stained with $5 \mathrm{mg} / \mathrm{mL}$ propidium iodide (PI) in PBS supplemented with RNase A (Roche, Indianapolis, IN, USA) for 30 minutes at room temperature. Cells in each individual phase of the cell cycle were determined based on their DNA ploidy profile using ACEA Flow Cytometer. The apoptosis rate was determined using Annexin V/PI staining kit from BD Biosciences.

\section{Immunofluorescence}

To determine subcellular distribution of mitochondria, cells were loaded with $50 \mathrm{nM}$ MitoTracker green (Life Technologies, Carlsbad, CA, USA) for 30 minutes to stain the mitochondria. Nuclei were counterstained with DAPI. Images were taken using Olympus FV1000 confocal microscope (Olympus, Tokyo, Japan).

\section{Statistical analysis}

SPSS version 16 (SPSS Inc, Chicago, USA) was used in this study. The $\chi^{2}$ test was used to check the correlations between Tbx2 and clinical parameters. The Kaplan-Meier method was used to estimate the probability of patient survival, and differences in the survival of subgroups of patients were compared by using Mantel's log-rank test. The Cox regression model was used for multivariate analysis. Student's $t$-test was used to compare differences between transfection and control groups. $P<0.05$ was considered to be significant.

\section{Results}

\section{Tbx2 is overexpressed in human} astrocytoma and confers poor prognosis

We examined expression of Tbx 2 protein in 102 astrocytoma specimens and 16 normal tissues using immunohistochemistry. In normal brain tissues, Tbx 2 expression was negative in astrocytes and neurons (Figure 1A). Positive nuclear Tbx2 staining was observed in 41 of 102 astrocytoma specimens (Figure 1B-D). We analyzed the relationship between clinical factors and Tbx2 status and found that high Tbx2 expression did not correlate with age or gender. Positive rate of Tbx2 protein in Grade I (pylocytic astrocytoma), Grade II (diffuse astrocytoma), Grade III (anaplastic astrocytoma), and Grade IV astrocytoma (glioblastoma multiform) were $0 \%, 26.1 \%, 40 \%$, and $52 \%$, respectively (Table 1 ). Glioblastoma multiform (GBM) showed significant higher positive rate compared with Grade I-III astrocytomas $(P=0.0327)$ (Table 1). Kaplan-Meier analysis showed that the patients with lower level of Tbx2 had longer overall survival (Figure 1E, $P<0.001$ ). Univariate and multivariate analyses revealed that $\mathrm{Tbx} 2$ was an independent predictor for overall survival of patients with astrocytoma of all grades (Table 2). 

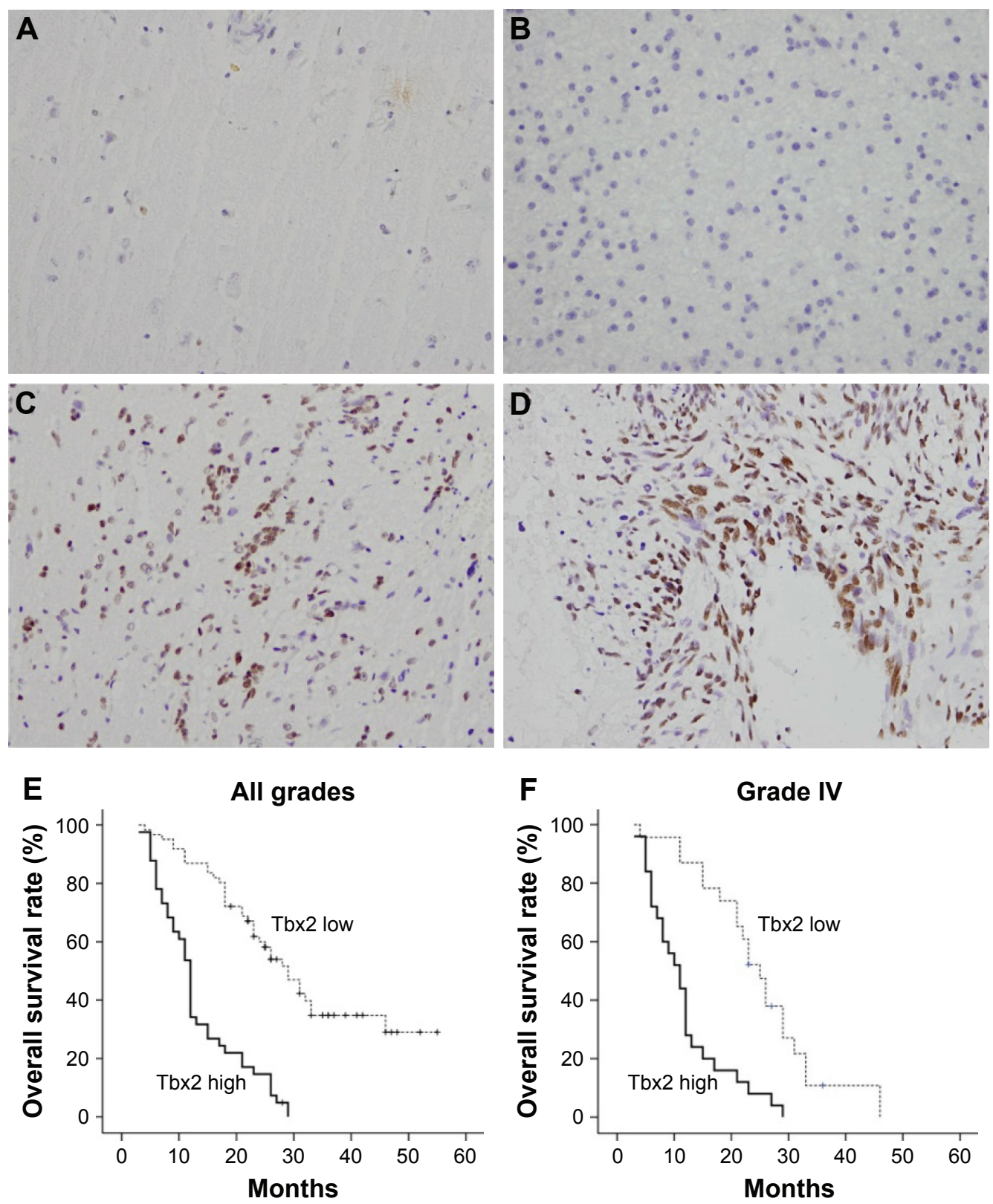

Figure I Expression pattern of Tbx2 in human astrocytoma.

Notes: (A) Immunohistochemical staining of Tbx2 protein in normal brain tissue was negative in glial cells and weak in neurons. (B) Negative Tbx2 staining in Grade II astrocytoma. (C) Positive nuclear Tbx2 staining in Grade III astrocytoma. (D) Positive nuclear Tbx2 staining in GBM (Grade IV). (E) Overall survival of patients with all grades of astrocytoma. (F) Overall survival of patients with GBM (Grade IV). Magnification 400x.

Abbreviation: GBM, glioblastoma multiform.

We also verified the prognostic value of Tbx2 in GBM group. Kaplan-Meier analysis showed that in GBM patients, those with high Tbx2 showed poorer survival than those with low Tbx2 (Figure 1F, $P<0.001$ ).

\section{Tbx2 promotes proliferation, invasion in GBM cell lines}

We examined protein expression of Tbx 2 in $3 \mathrm{GBM}$ cell lines (U87, U251 and A172) using Western blot and quantitative RT-PCR (RT-qPCR). We found high Tbx2 expression in
U251 cell line and low Tbx2 in U87 and A172 cell lines (Figure 2A). In order to explore the biological function of Tbx2 in astrocytoma cells, siRNA knockdown of Tbx2 was performed in $\mathrm{U} 251$ cell line, while Tbx2 plasmid transfection was carried out in A172 cell line. As shown in Figure 2B, the level of Tbx2 was significantly inhibited after siRNA treatment, and plasmid transfection upregulated Tbx 2 expression at both mRNA and protein levels.

We carried out CCK8 assay to examine the effect of Tbx 2 on cell proliferation. Tbx 2 overexpression upregulated cell 
Table I The relationship between Tbx2 and clinical features of astrocytoma

\begin{tabular}{lllll}
\hline Parameters & Number & \multicolumn{2}{l}{ Tbx2 } & P-value \\
\cline { 3 - 4 } & & Negative & Positive & \\
\hline Age & & & & 0.4267 \\
$\quad<50$ & 62 & 39 & 23 & \\
$\quad \geq 50$ & 40 & 22 & 18 & \\
Gender & & & & 0.7687 \\
Female & 29 & 18 & 11 & \\
Male & 73 & 43 & 30 & \\
Grading & & & & 0.0327 \\
Grade I & 6 & 6 & 0 & \\
Grade II & 23 & 17 & 6 & \\
Grade III & 25 & 15 & 10 & \\
Grade IV & 48 & 23 & 25 & \\
\hline
\end{tabular}

growth rate, while $\mathrm{Tbx} 2$ depletion inhibited cell proliferation (Figure 2C). In addition, we compared the cell growth rate in these three cell lines with different Tbx 2 status. As shown in Figure 2C, U251 with high endogenous Tbx2 exhibited highest growth rate. Then, we performed Matrigel invasion assay to analyze the potential effect of Tbx2 on cell invasion. As shown in Figure 2D, Tbx2 siRNA inhibited cell invasion in U251 cell line (control siRNA versus Tbx2 siRNA U251: $125.6 \pm 6.4$ versus $51 \pm 5.5, P<0.05$ ), while Tbx 2 overexpression upregulated invading ability in A172 cell line (empty vector versus Tbx2 plasmid A172: 64.6 \pm 4.7 versus $117 \pm 7.2, P<0.05)$. Using Western blot, we also found that $\mathrm{Tbx} 2$ overexpression upregulated protein expression of MMP2 and MMP9. Tbx2 depletion in U251 cell line inhibited MMP2 and MMP9 protein (Figure 3C). Altogether, these results demonstrated that $\mathrm{Tbx} 2$ could modulate both proliferation and invasion.

\section{Tbx2 promotes cell cycle progression in GBM cell lines}

To find out potential mechanism of Tbx2-induced proliferation, cell cycle analysis using flow cytometry was carried out. The results showed that Tbx2 transfection facilitated

Table 2 Univariate and multivariate analysis for predictive factors in patients with astrocytoma (Cox regression model)

\begin{tabular}{|c|c|c|c|c|}
\hline \multirow[t]{2}{*}{ Factors } & \multicolumn{2}{|l|}{ Univariate } & \multicolumn{2}{|l|}{ Multivariate } \\
\hline & $\begin{array}{l}\text { Hazard ratio } \\
(95 \% \mathrm{Cl})\end{array}$ & $P$-value & $\begin{array}{l}\text { Hazard ratio } \\
(95 \% \mathrm{Cl})\end{array}$ & $P$-value \\
\hline Age & $0.99(0.63-1.59)$ & 0.99 & $1.03(0.64-1.66)$ & 0.903 \\
\hline Gender & $1.20(0.73-1.97)$ & 0.468 & $1.35(0.82-2.28)$ & 0.243 \\
\hline Grade & $1.73(1.39-2.25)$ & $<0.001$ & $1.61(1.23-2.11)$ & $<0.001$ \\
\hline Tbx2 & $4.49(2.73-7.36)$ & $<0.001$ & $3.87(2.35-6.39)$ & $<0.001$ \\
\hline
\end{tabular}

Abbreviation: $\mathrm{Cl}$, confidence interval.
G1-S transition in A172 cells, with upregulation of S phase percentage and downregulation of G1 phase percentage. Tbx2 siRNA in U251 cells blocked G1-S cell cycle progression (Figure 4A). In addition, examination of cell cycle-related proteins demonstrated that $\mathrm{Tbx} 2$ overexpression increased cyclin D1 expression and decreased $\mathrm{p} 21$ protein. Tbx2 depletion downregulated cyclin D1, while it upregulated p21 (Figure 3C).

\section{Tbx2 reduces temozolomide-induced apoptosis in GBM cell lines}

The role of Tbx2 on apoptosis and resistance to chemotherapeutic drugs has not been investigated yet in GBM. We used temozolomide (TMZ) $(50 \mu \mathrm{M})$ to treat $\mathrm{U} 251$ and A172 cells transfected with Tbx2 siRNA or plasmid for 24,48 , and 72 hours. CCK- 8 assay showed a remarkable decrease in U251 cell viability after siRNA treatment. Tbx2 transfection significantly upregulated cell viability in A172 cells treated with TMZ (Figure 4B). We used Annexin V/PI staining to determine the changes in apoptosis after 24 hours of TMZ treatment. As shown in Figure 5A, Tbx2 overexpression reduced the rate of apoptosis induced by TMZ. Tbx 2 depletion increased apoptosis percentage in U251 cell line. In addition, Western blot demonstrated that Tbx 2 overexpression downregulated cleaved caspase 3 and upregulated caspase 3 . Tbx2 also downregulated cytochrome $\mathrm{C}$ and upregulated Bcl-2 expression. Tbx 2 depletion in U251 cells showed the opposite results (Figure 5B).

\section{Tbx2 regulates mitochondrial fission/ fusion and related proteins}

To investigate whether Tbx2 is involved in the dynamic balance of mitochondria, we examined mitochondrial morphology of GBM cells 48 hours after plasmid or siRNA transfection using confocal microscopy. Compared with controls, mitochondria in cells with Tbx 2 overexpression were more prone to fission (short mitochondria), while in U251 cells treated with Tbx2 siRNA, mitochondria were more prone to fusion (elongated shape) (Figure 3A). Then, we checked the change of mtDNA content. The mean mtDNA content increased in A172 cells with Tbx 2 overexpression. In contrast, mtDNA content decreased after Tbx 2 depletion in U251 cells (Figure 3B). Furthermore, we examined the level of p-Drp1(S616), the active form of Drp1, which promotes recruitment of Drp1 to the mitochondrial membrane, which induces increased mitochondrial fission. As shown in Figure 3C, Tbx2 overexpression upregulated p-Drp1, while Tbx2 depletion downregulated p-Drp1. 

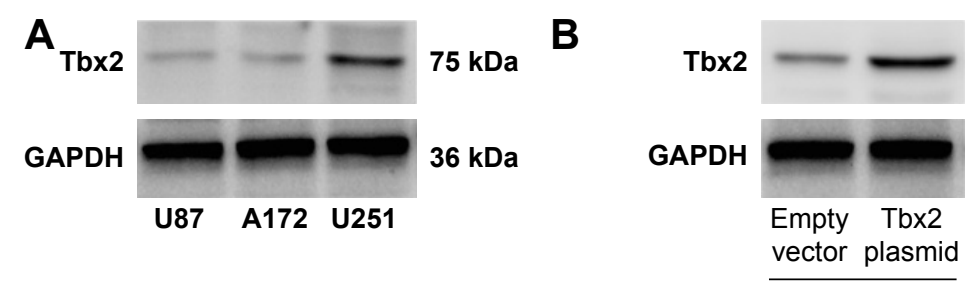

A172

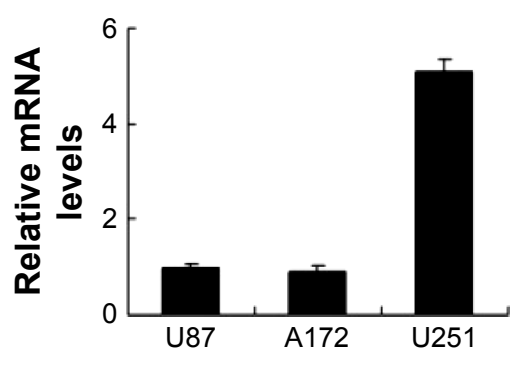

C

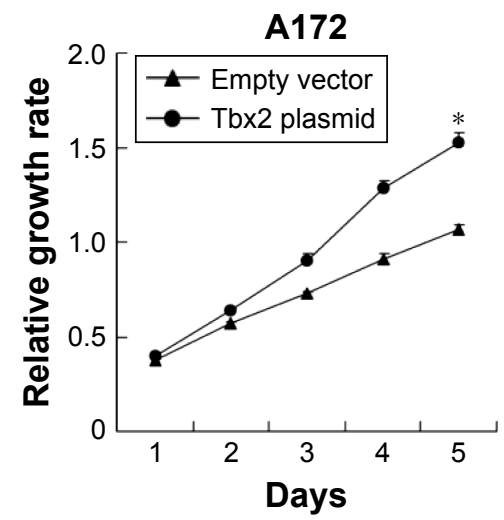

D

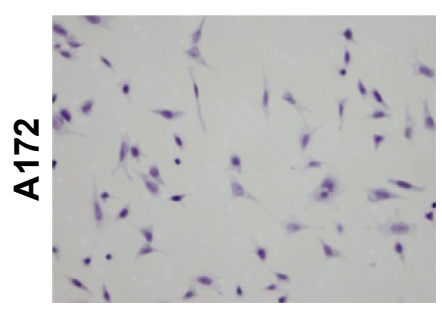

Empty vector

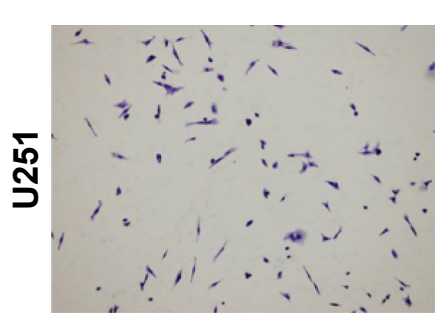

Control siRNA

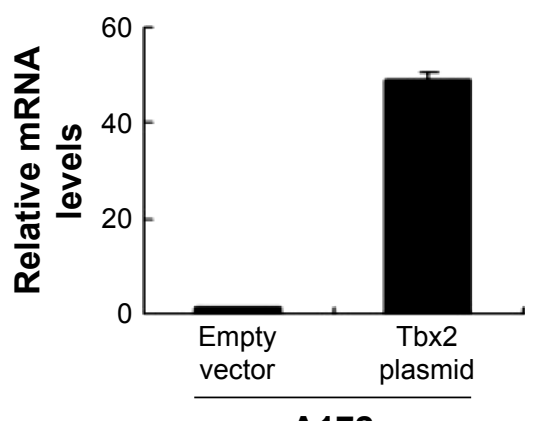

A172
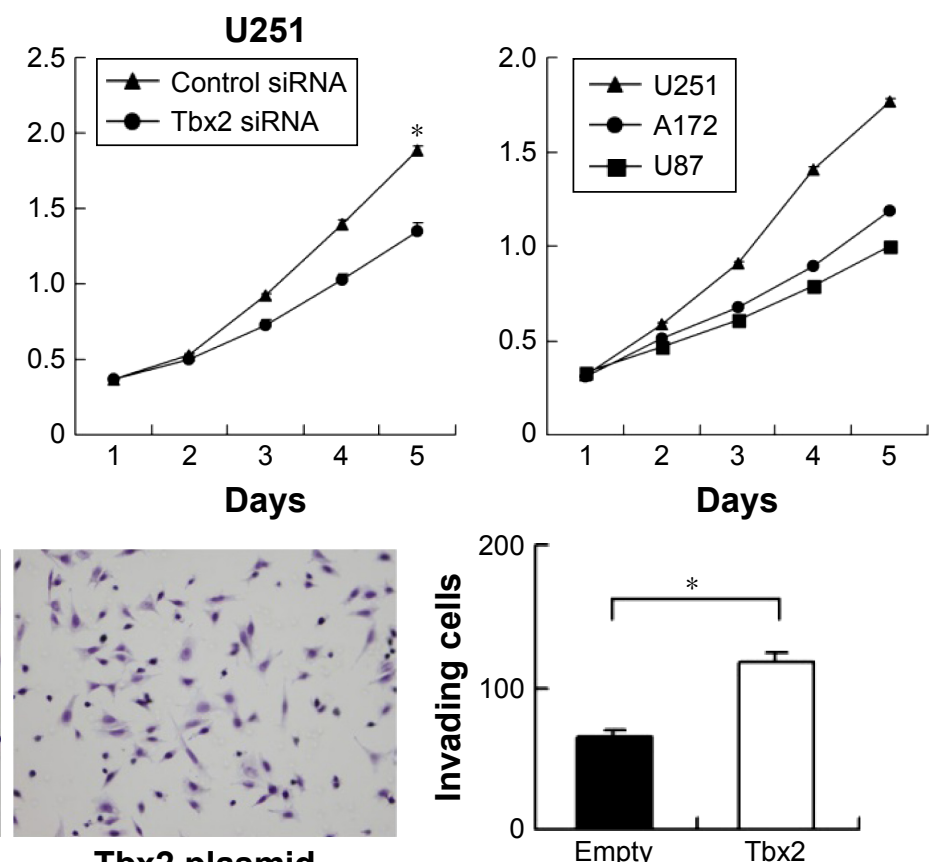

Tbx2 plasmid
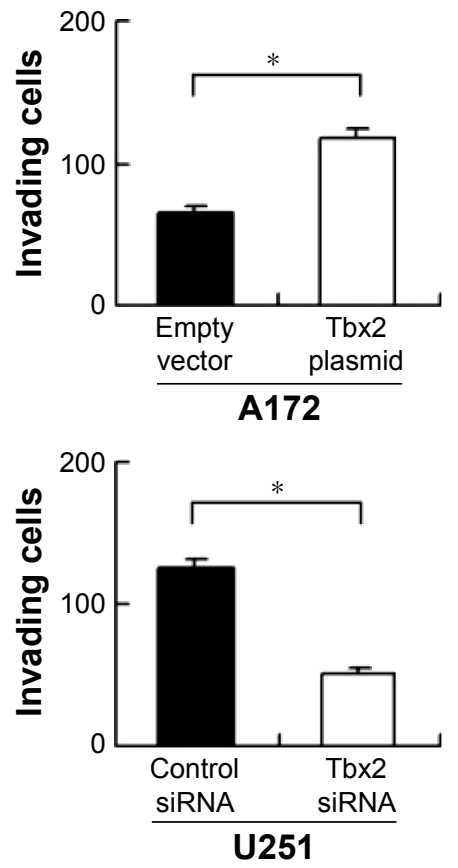

Figure 2 Tbx2 overexpression correlates with proliferation and invasion.

Notes: (A) Western blot and RT-PCR analysis showed that Tbx2 protein expression was high in U25I cell line and low in U87 and AI 72 cell lines. (B) Tbx2 siRNA treatment decreased its mRNA and protein expression in U25I cells. Tbx2 plasmid transfection upregulated its mRNA and protein levels. (C) CCK8 assay demonstrated that Tbx2 overexpression accelerated cell growth rate, while Tbx2 depletion inhibited cell proliferation. Cell growth rate was higher in U25I cell line than other cell lines. (D) Matrigel invasion assay showed that Tbx2 depletion decreased cell invasion in U25I cell line. Tbx2 plasmid transfection increased the number of invading cells. $* P<0.05$. Abbreviations: RT-PCR, real-time polymerase chain reaction; CCK8, Cell Counting Kit-8. 


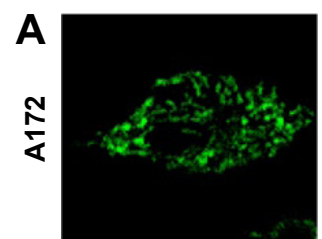

Empty vector

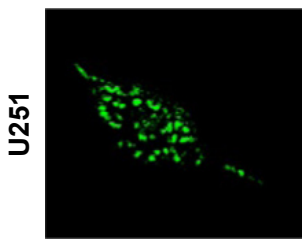

Control siRNA

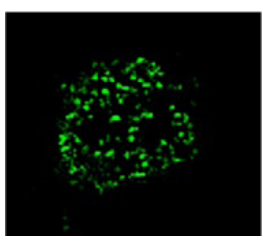

Tbx2 plasmid

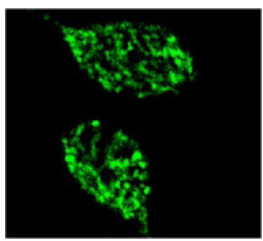

Tbx2 siRNA
B

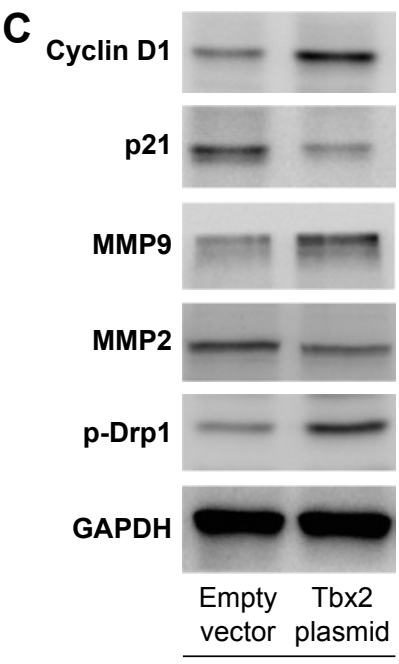

A172
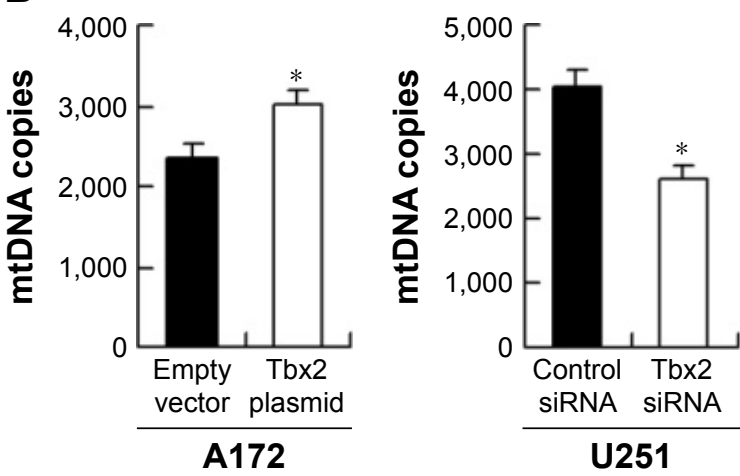

Figure 3 Tbx2 regulates mitochondrial fission/fusion and related proteins.

Notes: (A) Mitochondrial morphology was evaluated by confocal microscope using MitoTracker Green staining. Mitochondrial dynamics were prone to fission after Tbx2 overexpression in AI72 cells. Tbx2 depletion led to mitochondrial fusion in U25I cells. (B) Tbx2 overexpression upregulated mean mtDNA content in AI 72 cells with Tbx2 plasmid. In contrast, Tbx2 depletion decreased mtDNA content in U25I cells. (C) Tbx2 overexpression upregulated cyclin DI, MMP9, p-DrpI, and MMP2 and downregulated P2I in AI72 cell line. Tbx2 depletion downregulated cyclin DI, MMP9, p-DrpI, and MMP2 and upregulated p2I in U25I cell line. *P<0.05.

Abbreviation: mtDNA, mitochondrial DNA.

\section{Discussion}

In the present study, we demonstrated that Tbx 2 overexpression correlated with advanced grade and poor prognosis in human astrocytomas. We also showed that Tbx2 facilitated GBM cell proliferation and invasion and inhibited TMZinduced apoptosis. Tbx2 also modulated mitochondrial dynamics in GBM cell lines.

Tbx2 is a member of T-box transcription factor family containing DNA binding domain known as the T-box, which play a role in cell cycle progression and cancer development. In primary fibroblasts or striatal cells, overexpression of Tbx 2 can inhibit senescence through repression of ARF and p21 promoters, ${ }^{9}$ suggesting its oncogenic potential. Tbx 2 protein expression is elevated in several cancer tissues including lung, breast, and colorectal cancer and serves as a prognostic biomarker. ${ }^{4,7,8} \mathrm{Tbx} 2$ overexpression promotes cancer cell growth, invasion, and metastasis. ${ }^{4,5,10}$ However, the involvement of Tbx2 in astrocytoma development, especially in GBM, has not been explored. In this study, we showed elevated Tbx2 expression in $40.2 \%$ astrocytoma samples. Tbx2 protein staining was negative in normal glial cells and neurons. The rate of Tbx 2 overexpression was higher in Grade IV astrocytoma (glioblastoma multiform) than that in low-grade tumors, suggesting its correlation with malignant phenotype. Importantly, we demonstrated Tbx2 correlated with poor prognosis in patients with all-grade astrocytomas and in patients with GBM alone. Additionally, Tbx2 served as a prognostic factor, suggesting its expression status could be proposed for diagnostic or prognostic determination and therapeutic intervention.

The investigation of its biological effects in cell lines is a prerequisite to understand the roles of Tbx2 in GBM 
A

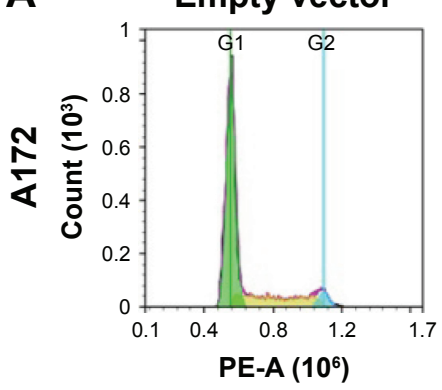

Control siRNA

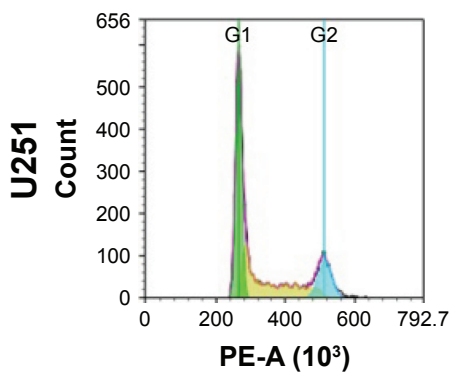

Tbx2 plasmid

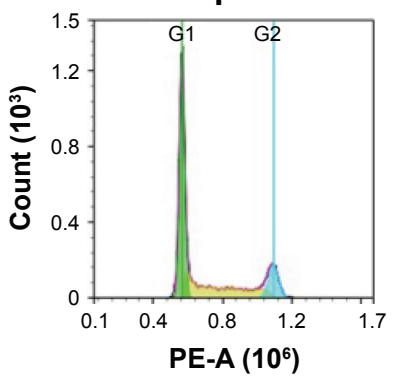

Tbx2 siRNA

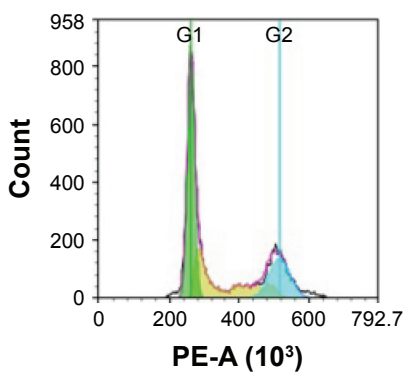

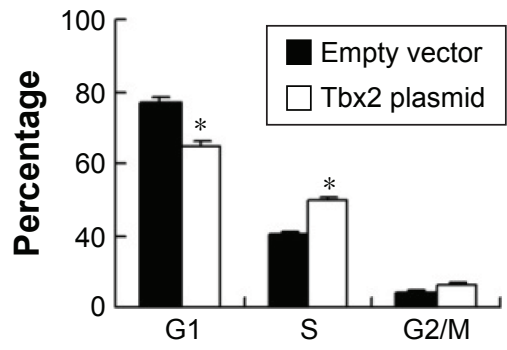

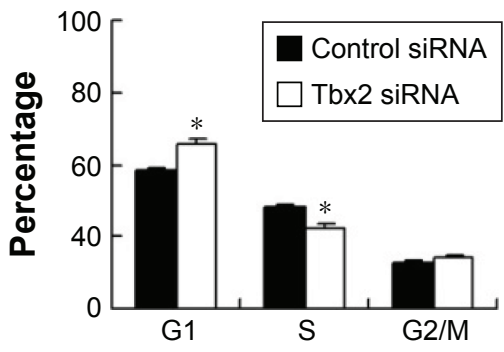

B

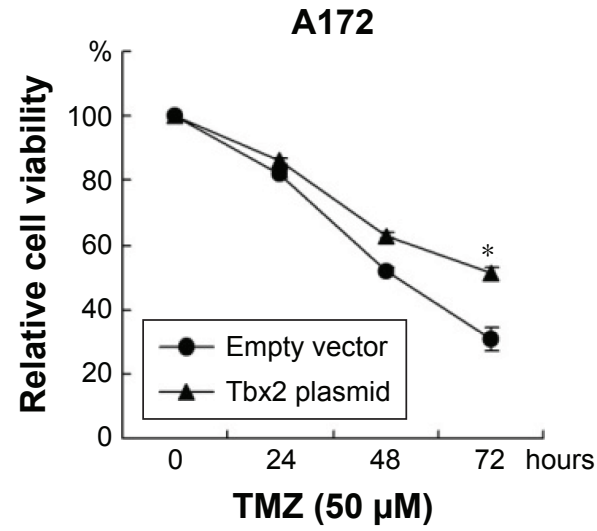

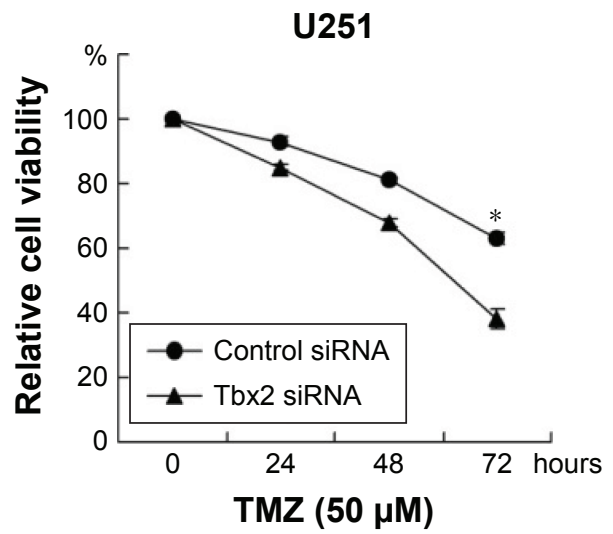

Figure 4 Tbx2 regulates cell cycle and TMZ resistance in GBM cell lines.

Notes: (A) Cell cycle analysis showed that Tbx2 overexpression increased the proportion of cells in $S$ phase. Tbx2 depletion downregulated the proportion of cells in $\mathrm{S}$ phase. (B) CCK8 assay showed that Tbx2 depletion decreased cell viability with TMZ $(50 \mu \mathrm{M})$ treatment. Tbx2 overexpression upregulated cell viability with TMZ (50 $\mu \mathrm{M})$ treatment. $* P<0.05$.

Abbreviations: GBM, glioblastoma multiform; TMZ, temozolomide; CCK8, Cell Counting Kit-8.

progression. We transfected Tbx2 siRNA and plasmid in U251 and A172 cell lines, respectively. Decreased proliferation and invading ability was observed after Tbx2 knockdown in U251 cell line. Tbx2 overexpression facilitated cell proliferation and invasion in A172 cell line. These findings indicated that Tbx2 might play an important role during malignant progression of astrocytoma cells. Cell cycle analysis showed that Tbx2 facilitated cell cycle progression. We checked several cell cycle-related proteins and found that Tbx2 upregulated cyclin D1 and downregulated p21. Cyclin D1 positively regulates G1-S progression. ${ }^{11}$ Activation of cyclin D1 has been demonstrated in human astrocytomas. ${ }^{12-14} \mathrm{p} 21$ is a cyclin-dependent kinase inhibitor that is downregulated in astrocytoma. ${ }^{15}$ It has been reported that $\mathrm{Tbx} 2$ directly represses the expression of the $\mathrm{p} 21$ promoter in vitro and in vivo., ${ }^{916}$ Together, these results indicated that $\mathrm{Tb} x 2$ facilitated malignant cell growth through regulation of cell cycle-related proteins. Next, we checked invasionrelated factors and found that $\mathrm{Tbx} 2$ positively regulated MMP2 and MMP9, both of which are capable of degrading extracellular matrix proteins, facilitating GBM invasion. ${ }^{17,18}$ A previous study reported that Tbx 2 could induce epithelialmesenchymal transition. ${ }^{19}$ However, astrocytoma is a nonepithelial cancer without E-cadherin. Thus, we assumed that EMT was not responsible for Tbx2-induced cell invasion.

The role of Tbx 2 on chemoresistance and apoptosis of GBM cells remains unclear. To clarify this issue, we examined the change of cell viability and apoptosis after TMZ 
A

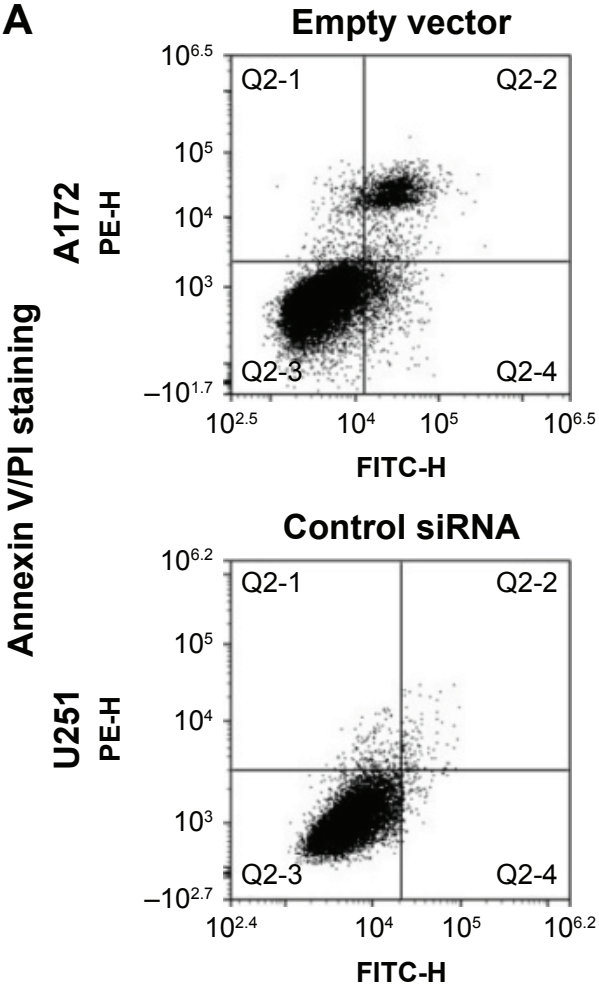

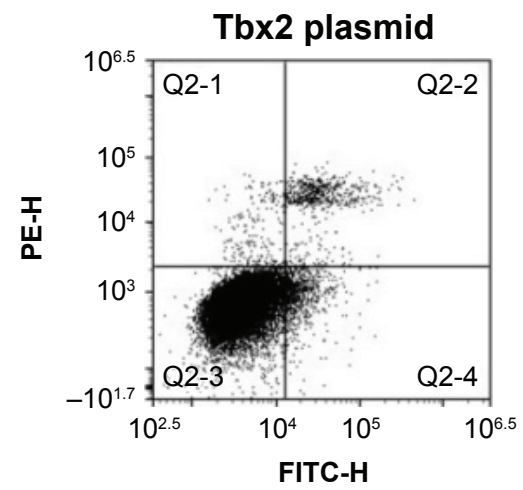

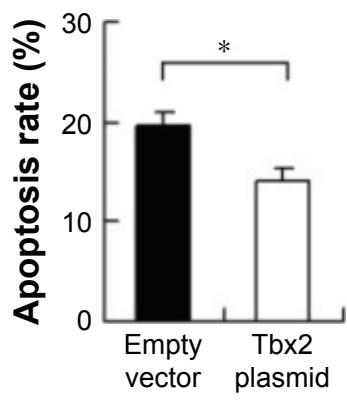

A172
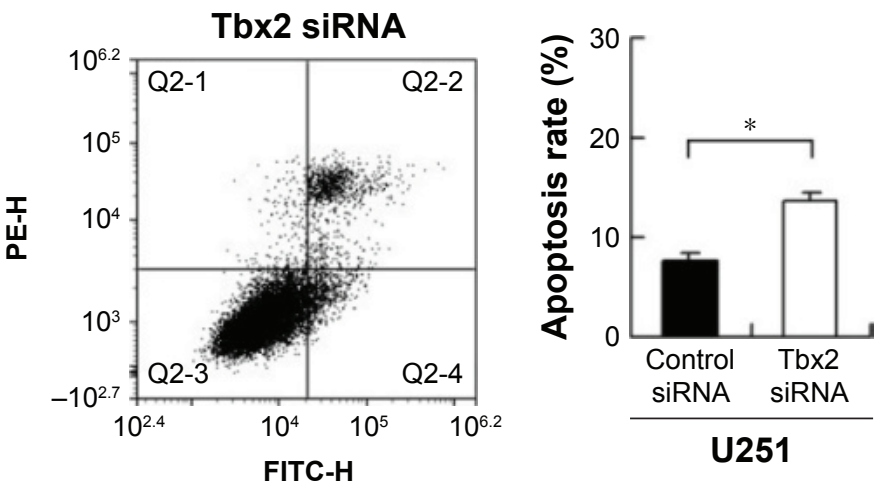

B

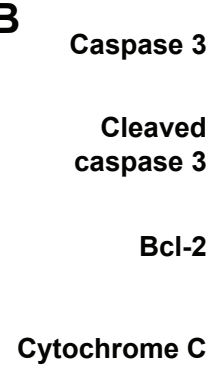

GAPDH
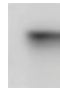

d
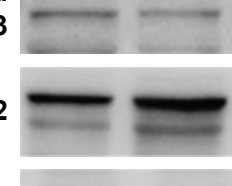

C

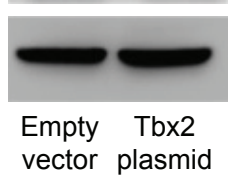

A172

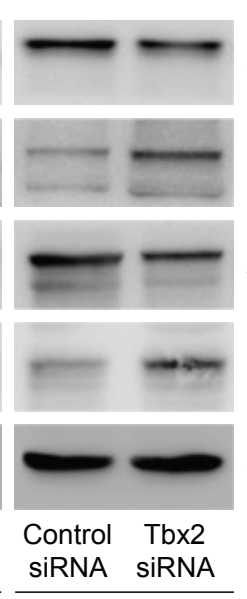

U251
$35 \mathrm{kDa}$

$17 \mathrm{kDa}$

$28 \mathrm{kDa}$

$15 \mathrm{kDa}$

$36 \mathrm{kDa}$

Figure 5 Tbx2 alleviated TMZ-induced apoptosis in GBM cell lines.

Notes: (A) Annexin V/PI staining and flow cytometry analysis showed that Tbx2 downregulated apoptosis rate induced by TMZ treatment in AI72 cells. Tbx2 siRNA upregulated apoptosis induced by TMZ in U25I cells. (B) Tbx2 overexpression upregulated Bcl-2 and caspase 3 and downregulated cleaved caspase 3 and cytochrome $C$ in Al72 cell line. Tbx2 depletion downregulated $\mathrm{Bcl}-2$ and caspase 3 and upregulated cleaved caspase 3 and cytochrome $\mathrm{C}$ in $\mathrm{U} 25 \mathrm{I}$ cell line. ${ }^{*} \mathrm{P}<0.05$.

Abbreviations: GBM, glioblastoma multiform; PI, propidium iodide; TMZ, temozolomide.

treatment. Tbx2 depletion sensitized cancer cells to TMZ treatment by upregulation of apoptosis rate and downregulation of cell viability. Tbx2 reduced TMZ-induced apoptosis. Accordingly, Tbx2 overexpression reduced the level of caspase 3 cleavage, indicating decreased level of apoptosisinduced cell death. In addition, Tbx2 upregulated Bcl-2 while reducing cytochrome $\mathrm{C}$ level. Bcl-2 plays an important role during development of chemoresistance in GBM and correlates with poor patient prognosis. ${ }^{20-22}$ Cytochrome C serves as an indicator of mitochondria-related apoptosis. ${ }^{23}$ Thus, these results suggest the role of Tbx 2 as an inhibitor of apoptosis.

Mitochondrial morphology is regulated by the balance between fusion and fission, which is controlled by dynamin-related GTPases. ${ }^{24}$ Mitochondrial dynamics have recently been associated with malignant activity of cells. It was reported that increased mitochondrial fission promoted the survival of cancer cells by autophagy and inhibition 
of mitochondria dependent apoptosis. ${ }^{25}$ Mitochondrial hyperfusion sensitizes human cancer cells to drug-induced apoptosis. ${ }^{26}$ Our results showed that Tbx2 induced mitochondrial fission, which contributes to the survival of GBM cells. Accordingly, we found upregulation of p-Drp1 after Tbx2 overexpression. Drp1 phosphorylation at ser 616 promotes recruitment of Drp1 to the mitochondrial membrane, which induces increased mitochondrial fission. ${ }^{27}$ To better define the change of mitochondria function, we examined mtDNA content. mtDNA plays a central role in maintaining normal mitochondrial function, and many studies indicated that changes in mtDNA content may contribute to cancer progression. ${ }^{28}$ We found that Tbx2 overexpression upregulated mtDNA content and that its depletion downregulated mtDNA content, suggesting Tbx2 maintains normal mitochondrial function in astrocytoma cell lines. Taken together, these results indicate Tbx2 alleviates mitochondria dysfunction and disruption in GBM cell lines.

In conclusion, $\mathrm{Tbx} 2$ is overexpressed in human astrocytoma and is correlated with advanced grade and poor survival. Tbx2 promotes GBM cell growth and invasion and inhibits TMZ-induced apoptosis, possibly through regulation of mitochondrial dynamics. Based on these findings, Tbx2 might serve as a useful biomarker and therapeutic target in human astrocytoma.

\section{Disclosure}

The authors report no conflicts of interest in this work.

\section{References}

1. Siegel R, Naishadham D, Jemal A. Cancer statistics, 2012. CA Cancer J Clin. 2012;62(1):10-29.

2. Krupkova O Jr, Loja T, Redova M, et al. Analysis of nuclear nestin localization in cell lines derived from neurogenic tumors. Tumour Biol. 2011;32(4):631-639.

3. Zadran S, Amighi A, Otiniano E, Wong K, Zadran H. ENTPD5-mediated modulation of ATP results in altered metabolism and decreased survival in gliomablastoma multiforme. Tumour Biol. 2012;33(6):2411-2421.

4. Douglas NC, Papaioannou VE. The T-box transcription factors TBX2 and TBX3 in mammary gland development and breast cancer. J Mammary Gland. 2013;18(2):143-147.

5. Abrahams A, Parker MI, Prince S. The T-box transcription factor Tbx2: its role in development and possible implication in cancer. IUBMB Life. 2010;62(2):92-102.

6. D'Costa ZC, Higgins C, Ong CW, et al. TBX2 represses CST6 resulting in uncontrolled legumain activity to sustain breast cancer proliferation: a novel cancer-selective target pathway with therapeutic opportunities. Oncotarget. 2014;5(6):1609-1620.

7. Hu B, Mu HP, Zhang YQ, et al. Prognostic significance of TBX2 expression in non-small cell lung cancer. J Mol Histol. 2014;45(4): 421-426.

8. Han Y, Tu WW, Wen YG, et al. Increased expression of TBX2 is a novel independent prognostic biomarker of a worse outcome in colorectal cancer patients after curative surgery and a potential therapeutic target. Med Oncol. 2013;30(4):688.
9. Prince S, Carreira S, Vance KW, Abrahams A, Goding CR. Tbx2 directly represses the expression of the $\mathrm{p} 21$ (WAF1) cyclin-dependent kinase inhibitor. Cancer Res. 2004;64(5):1669-1674.

10. Zhang Z, Guo Y. High TBX2 expression predicts poor prognosis in non-small cell lung cancer. Neoplasma. 2014;61(4):476-480.

11. Yang J, Yang Q, Yu J, Li X, Yu S, Zhang X. SPOCK1 promotes the proliferation, migration and invasion of glioma cells through $\mathrm{PI} 3 \mathrm{~K} /$ AKT and Wnt/beta-catenin signaling pathways. Oncol Rep. 2016;35: 3566-3576.

12. Jiao H, Guan F, Yang B, et al. Human umbilical cord blood-derived mesenchymal stem cells inhibit C6 glioma via downregulation of cyclin D1. Neurol India. 2011;59(2):241-247.

13. Abdullah JM, Ahmad F, Ahmad KA, et al. Molecular genetic analysis of BAX and cyclin D1 genes in patients with malignant glioma. Neurol Res. 2007;29:239-242.

14. Arato-Ohshima T, Sawa H. Over-expression of cyclin D1 induces glioma invasion by increasing matrix metalloproteinase activity and cell motility. Int J Cancer. 1999;83:387-392.

15. Liu J, Estes ML, Drazba JA, et al. Anti-sense oligonucleotide of p21(waf1/cip1) prevents interleukin 4-mediated elevation of p27(kip1) in low grade astrocytoma cells. Oncogene. 2000;19:661-669.

16. Huang Y, Li Z, Zhong Q, Li G, Zhang Y, Huang Z. Association of TBX2 and P21 expression with clinicopathological features and survival of laryngeal squamous cell carcinoma. Int $J$ clin Exp Med. 2014;7:5394-5402.

17. Zhu X, Morales FC, Agarwal NK, Dogruluk T, Gagea M, Georgescu MM. Moesin is a glioma progression marker that induces proliferation and Wnt/beta-catenin pathway activation via interaction with CD44. Cancer Res. 2013;73:1142-1155.

18. Tabouret E, Boudouresque F, Farina P, et al. MMP2 and MMP9 as candidate biomarkers to monitor bevacizumab therapy in high-grade glioma. Neuro Oncol. 2015;17:1174-1176.

19. Wang B, Lindley LE, Fernandez-Vega V, Rieger ME, Sims AH, Briegel KJ. The T box transcription factor TBX2 promotes epithelialmesenchymal transition and invasion of normal and malignant breast epithelial cells. PLoS One. 2012;7:e41355.

20. McDonald FE, Ironside JW, Gregor A, et al. The prognostic influence of bcl-2 in malignant glioma. Br J Cancer. 2002;86:1899-1904.

21. Fels C, Schafer C, Huppe B, et al. Bcl-2 expression in higher-grade human glioma: a clinical and experimental study. J Neurooncol. 2000; 48:207-216.

22. Wick W, Wagner S, Kerkau S, Dichgans J, Tonn JC, Weller M. BCL-2 promotes migration and invasiveness of human glioma cells. FEBS Lett. 1998;440:419-424.

23. Gao X, Deeb D, Jiang H, Liu YB, Dulchavsky SA, Gautam SC. Curcumin differentially sensitizes malignant glioma cells to TRAIL/Apo2Lmediated apoptosis through activation of procaspases and release of cytochrome c from mitochondria. $J$ Exp Ther Oncol. 2005;5(1): $39-48$.

24. Chan DC. Mitochondrial fusion and fission in mammals. Annu Rev Cell Dev Biol. 2006;22:79-99.

25. Huang Q, Zhan L, Cao H, et al. Increased mitochondrial fission promotes autophagy and hepatocellular carcinoma cell survival through the ROS-modulated coordinated regulation of the NFKB and TP53 pathways. Autophagy. 2016;12:999-1014.

26. Akita M, Suzuki-Karasaki M, Fujiwara K, et al. Mitochondrial division inhibitor-1 induces mitochondrial hyperfusion and sensitizes human cancer cells to TRAIL-induced apoptosis. Int J Oncol. 2014;45: 1901-1912.

27. Taguchi N, Ishihara N, Jofuku A, Oka T, Mihara K. Mitotic phosphorylation of dynamin-related GTPase Drp1 participates in mitochondrial fission. J Biol Chem. 2007;282:11521-11529.

28. Yu M. Generation, function and diagnostic value of mitochondrial DNA copy number alterations in human cancers. Life Sci. 2011;89:65-71. 
OncoTargets and Therapy

\section{Publish your work in this journal}

OncoTargets and Therapy is an international, peer-reviewed, open access journal focusing on the pathological basis of all cancers, potential targets for therapy and treatment protocols employed to improve the management of cancer patients. The journal also focuses on the impact of management programs and new therapeutic agents and protocols on

patient perspectives such as quality of life, adherence and satisfaction. The manuscript management system is completely online and includes a very quick and fair peer-review system, which is all easy to use. Visit http://www.dovepress.com/testimonials.php to read real quotes from published authors.

Submit your manuscript here: http://www.dovepress.com/oncotargets-and-therapy-journal 